\title{
The Research on Plant Information Acquisition Based on Hyperspectral Imaging Technique
}

\author{
Lei Shi ${ }^{1}$, Shengkun Yan ${ }^{2}$ \\ ${ }^{1}$ Xinjiang Institute of Engineering, Urumqi, China \\ ${ }^{2}$ Zhengzhou College of Traffic Technicians, Zhengzhou, Henan Province, China
}

Keywords: Spectrum, Spectral imaging, Imaging technology, Plant information

\begin{abstract}
As a new remote sensing technique, hyperspectral remote sensing has been widely used in agriculture and plants. Hyper spectroscopy can accurately detect and obtain the fine spectral information of plants to invert biochemical and physical parameters and carry out pest and disease monitoring, further develop and improve the remote sensing monitoring technology of plants, and greatly improve the monitoring efficiency and accuracy. Hyperspectral remote sensing has a certain application potential in plant growth monitoring. The application of hyperspectral remote sensing image to plant growth monitoring is an important direction for the rapid development and implementation of precision agriculture in the future.
\end{abstract}

\section{Introduction}

Spectral characteristics of plants are the result of the influence of environmental factors (biological factors and abiotic factors).The rapid and non-destructive acquisition of plant nutrient physiological information, the indirect prediction of plant yield and the monitoring of plant growth and response to stress by using spectral and imaging techniques are conducive to the realization of agriculturalization, digitization, informatization and intelligent management. Spectral imaging technology combines spectral analysis technology and imaging technology, it can obtain not only spectral information and spatial information of samples, but also the physical and chemical characteristics of samples. Spectral images are usually three-dimensional (3D), consisting of two-dimensional spatial information and one-dimensional spectral information. According to the number of bands, spectral imaging technology can be divided into multispectral imaging technology and hyperspectral imaging technology. In general, the image obtained by hyperspectral imaging consists of a large number of continuous bands (tens or hundreds), while the image obtained by multispectral imaging consists of a series of discrete bands (generally less than 10).

The spectral resolution of hyperspectral image is higher, the sample information can be obtained better, and the accuracy of monitoring plant information is higher. However, because hyperspectral images usually carry a large amount of information, it is necessary to reduce the dimension of the data and remove redundant information. Hyperspectral imaging technology also has its limitations, such as high cost and slow processing speed. Therefore, hyperspectral imaging technology is mainly used in basic research. Compared with hyperspectral imaging technology, multispectral imaging technology is more suitable for large-area field monitoring. At the same time, due to the progress of technology, the current near-field multispectral imaging technology and hyperspectral imaging technology are used in the study of plant phenotypes [1].

\section{Vegetation Index}

Vegetation index is a kind of combination of spectral values of different bands with certain biochemical significance, which usually includes ratio vegetation index, linear combination vegetation index, modified vegetation index, difference vegetation index, etc. The vegetation index of different band combinations has different prediction effects on different indexes. In agriculture, spectral techniques are generally used to detect the physiological index of plants in the band range of 
$400 \sim 2,500 \mathrm{~nm}$, involving the absorption of pigments (chlorophyll, carotenoids, etc.), nitrogen, water, and the internal structure of leaf cells. In the visible band of $400 \sim 740 \mathrm{~nm}$, chlorophyll has absorption peaks at 480, 650, $670 \sim 680$ and $740 \mathrm{~nm}$, carotenoids have absorption peaks at 420, 425, 440, 450, 470 and $480 \mathrm{~nm}$, and lutein has absorption peaks at 425, 445 and $475 \mathrm{~nm}$, shown as Fig.1. However, the reflectivity of healthy mesophyll cells increased sharply at $740 \sim 1,300 \mathrm{~nm}$ near infrared band. The absorption peaks of plant water were mainly at 970,1 450 and $1944 \mathrm{~nm}$. Therefore, when plants are under stress, their nitrogen, pigments and enzymes change accordingly. By monitoring the changes of these physiological indicators with various vegetation indexes, the stress, growth and yield of plants can be judged. However, there are only a few bands in the multispectral spectrum. Although some vegetation indexes can be constructed, the constructed vegetation indexes may not reflect the physiological and biochemical information and growth status of plants [2]. Hyper spectroscopy is different, with hundreds of bands of information, even the same vegetation index, there can be thousands of combinations, and so many combinations and so many vegetation index, can always find suitable for monitoring the physiological and biochemical information and growth state of plants sensitive index.

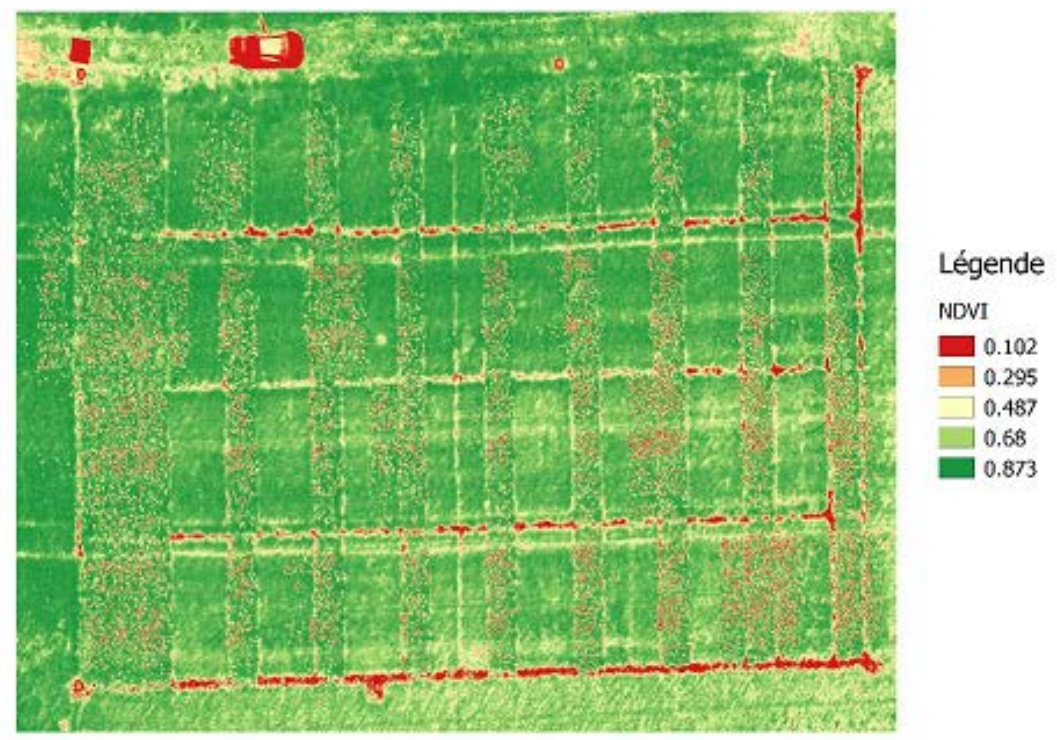

Fig.1 Plant Growth Profile in Uav Multi-Spectral Image

\section{Acquisition of Plant Information by Hyperspectral Imaging Technique}

\subsection{Nutrient Nitrogen Content Monitoring}

The nitrogen balance index (NBI) is one of the important indicators of plant growth. The real-time nondestructive monitoring of plant nitrogen $(\mathrm{N})$ is important for the accurate management of nitrogen in plant production. Using the hyperspectral remote sensing data can monitor the status of plant nitrogen fertilizer in real time, dynamically, non-destructively, quickly and effectively, provide accurate information for agricultural production and management, and guide farmers to apply nitrogen fertilizer accurately and rationally, so as to reduce farmers' cost and nitrogen pollution to the environment. Nitrogen and chlorophyll contents are important nutrient indexes of plants, as shown in Fig. 3, and are closely related to plant yield [3]. The acquisition of plant nutrient information based on spectroscopy and imaging technology can be divided into rapid acquisition of plant nutrient information based on direct spectral information (such as stepwise multiple regression, partial quadratic multiplication, weight coefficient, support vector machine, etc.) and rapid acquisition of plant nutrient information based on vegetation index according to whether the information is directly used or not. Plant nutrient acquisition based on direct spectral information is to detect plant nutrient information through original spectral processing modeling, while nutrient detection based on vegetation index is to establish vegetation index and nutrient model for analysis. 


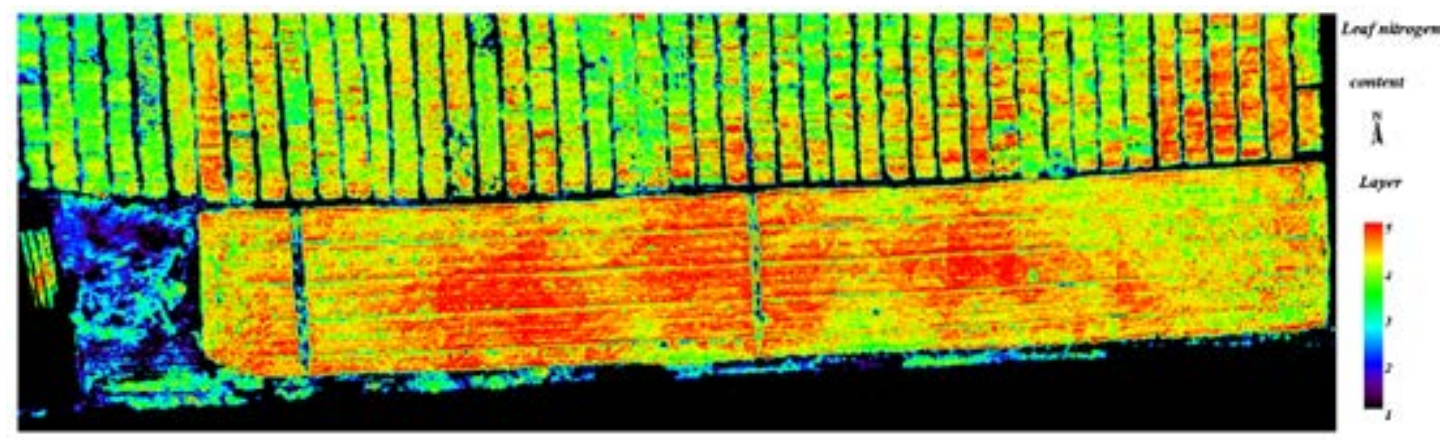

Fig.3 Aerial Hyperspectral Image of Plant Nitrogen Distribution

\subsection{Chlorophyll Content Monitoring}

Chlorophyll content is an important indicator of vegetation stress state and an important basis for estimation of other biochemical parameters [4]. Estimation of chlorophyll content by hyperspectral technique is of great significance for assessing plant growth status, predicting yield and monitoring growth, as shown in FIG. 3.It can provide a reference for estimating chlorophyll content of plant canopy and lay a foundation for real-time, rapid and nondestructive monitoring of plant chlorophyll content.

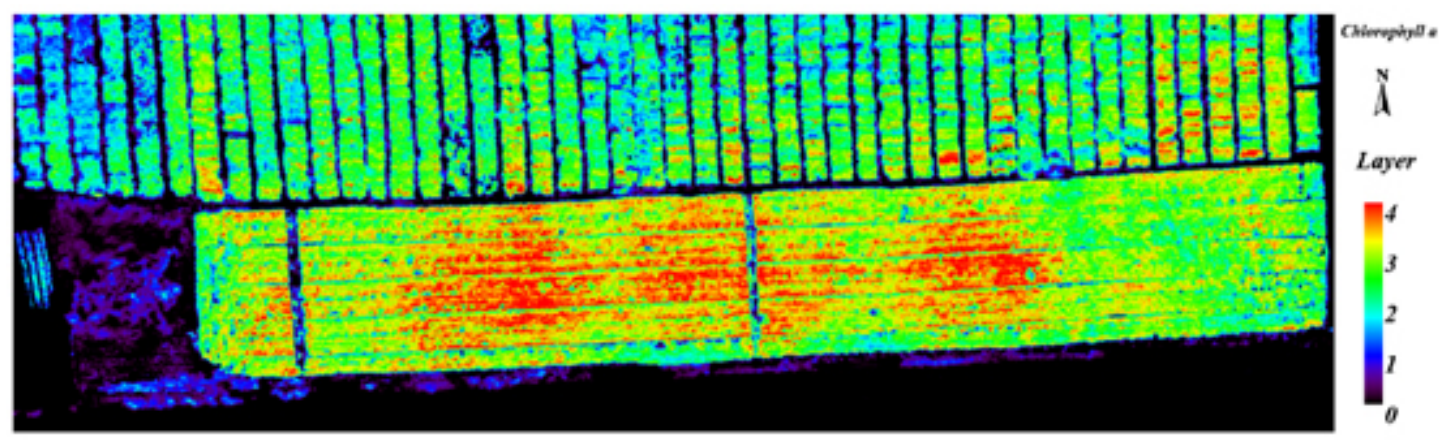

Fig.3 Aerial Hyperspectral Image of Plant Chlorophyll a Distribution

Sun et al. built the estimation model of chlorophyll mass fraction of winter wheat by using regression analysis method and BP neural network method respectively, and compared the estimation model with the measured chlorophyll mass fraction in the field, analyzed the inversion accuracy, and screened out the model with the highest accuracy. J. Lu et al. found that the application of particle swarm optimization algorithm and support vector mechanism to establish a leaf-scale hyperspectral estimation model for the chlorophyll content of maize could accurately predict the chlorophyll content of maize and solve the problem of chlorophyll content inversion in the case of small sample maize sampling points [5]. Using the hyperspectral reflectance of cotton leaves in flower-boll stage as the data source, Sh. Wang analyzed the correlation between the 13 planting target index and the optimized spectral index RSI and the relative content of chlorophyll in cotton leaves (SPAD), constructed the linear and nonlinear regression monitoring model and verified it. W.L. Zhang et al. selected Kandelia candel as the research object, collected leaves and measured the reflection spectrum and chlorophyll content of the front and back of leaves [6]. Thirteen commonly used parameters were selected for sensitivity analysis, and further parameters with higher chlorophyll correlation coefficient were selected to establish the estimation model.

\subsection{Water Stress Monitoring}

Plant water potential is an important physiological index to reflect the water status of plants. The monitoring of plant water potential can provide scientific basis for the accurate management of plant irrigation [6]. The traditional method of plant water potential monitoring is time-consuming, labor-intensive and limited to a small range of monitoring points. 
By using hyperspectral technique, the plant water potential can be estimated quickly ((the greater the WBI value, the higher the water content of the plant, the better for plant growth), shown as Fig.4, nondestructively and accurately, so as to optimize the irrigation scheme [7]. The rapid acquisition of plant water stress information by spectral and imaging techniques is beneficial to the chemical control of plant fertilizer and water management. X.D. Zhang et al. applied multi-spectral imaging and hyperspectral techniques to study the water content of rape leaves under water stress. The prediction results of the model based on hyper spectroscopy are better than those based on multispectral imaging [8].

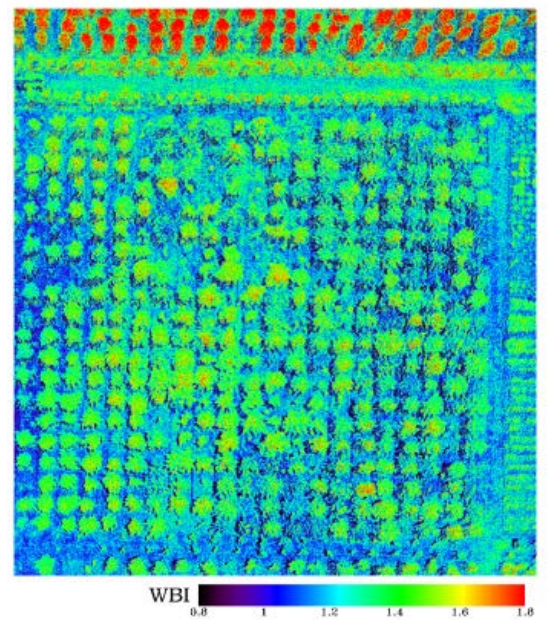

Fig.4 Change of Wbi Index

\subsection{Disease Stress Monitoring}

In the process of plant growth, pests and diseases will reduce its yield and quality, affecting the economic benefits. Monitoring and early warning of diseases and insect pests and adopting methods to control them can effectively improve plant yield and quality and reduce economic losses. However, the traditional plant pest monitoring method is not only inefficient, but also has the problems of low accuracy and low effectiveness [9]. Therefore, the advantages of accurate and real-time hyperspectral remote sensing make it an efficient means and cutting-edge technology for plant disease and insect pest monitoring.

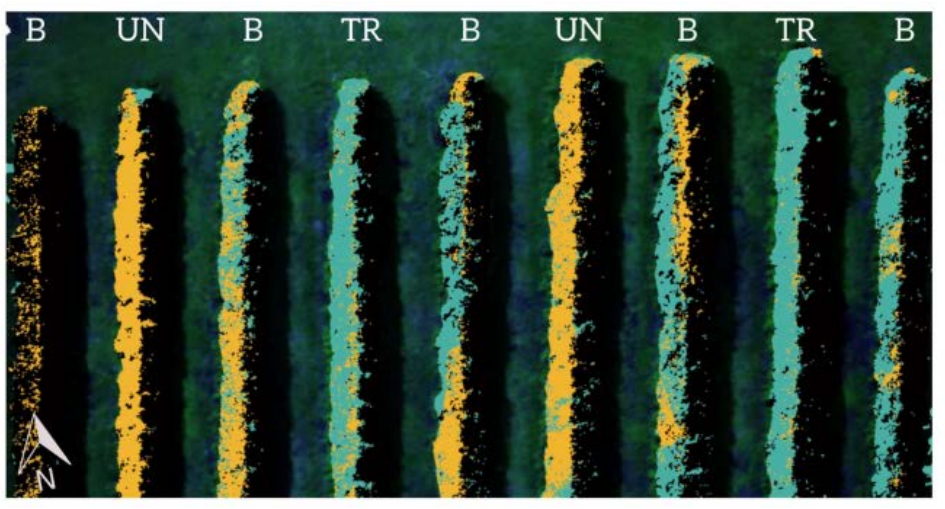

Fig.5 Disease Stress (the More Yellow the More Serious the Disease, the Less Green the Disease)

At present, the diagnosis of diseases and insect pests can be divided into direct and indirect methods. The direct direction is mainly chemical analysis methods, including polymerase chain reaction, DNA array and other methods. The indirect method is mainly the sensor technology of electronic nose, spectrometer and so on. Spectrum and imaging technology is a fast, non - destructive and effective technique for the diagnosis of diseases and insect pests. When plants are under the stress of diseases and insect pests, the internal physiological indicators and external morphology of plants will change, which are presented by spectral response, texture, color and other characteristics in spectral and imaging techniques [10]. Therefore, spectral and imaging techniques diagnose plant 
disease and insect pest stress by analyzing one or more spectral bands and plant image information. In addition, the vegetation index used to diagnose diseases and insect pests mainly includes normalized vegetation index, green normalized vegetation index, ratio vegetation index, photochemical reflection, leaf moisture vegetation index 1 , moisture index, and moisture band index, etc., as shown in Fig. 5.

\section{Summary}

The perception and acquisition of plant physiological information has played an important role in agricultural production, decision making and the detection of plant growth status, and has become an important part of the development of agriculture and agricultural informatization. In plant growth, plant nutrient physiology information fast condition (n, chlorophyll, protein, enzymes, etc.) of agricultural production is still the emphasis and difficulty in management and operations research, relevant methods and technology breakthrough, to actualize digital and information-based agriculture, and intelligent management and operation is of great significance.

\section{Acknowledgement}

Project: Colleges and Universities Scientific Research Project in Xinjiang Autonomous Region (Project Number: XJEDU2019Y066).

\section{References}

[1] W.L. Zhang, C.Sh. Zeng and D.Zh. Gao, Estimation of chlorophyll content by hyperspectral remote sensing in autumn solanum, Journal of ecology, vol. 21, pp. 90-93, 2014.

[2] Q.Y. Sun, Q.R. Chang and M.T. Liu, Hyperspectral remote sensing inversion of chlorophyll mass fraction in winter wheat canopy, Acta agriculturae boretum, vol. 4, pp. 55-57, 2017.

[3] J. Lu, K.G. Wang and Ch.G. Li, Estimation model of chlorophyll content of maize based on hyperspectral remote sensing, Journal of China university of mining and technology, vol. 2, pp. 40-41,2016.

[4] Sh. Wang, Estimation of chlorophyll content in cotton leaves based on hyperspectral remote sensing, Journal of China agricultural university, vol. 4, pp. 16-17,2017.

[5] Ch.Ch. Li, P. Chen and G.Zh. Lu, Inversion of nitrogen balance index in typical growth period of soybean based on high-resolution digital image of uav and hyperspectral remote sensing data, Journal of applied ecology, vol. 4, pp. 12-14,2018.

[6] Zh.F. Chen, N. Song and J.L. Wang, Estimation model of leaf water potential of winter wheat based on hyperspectral remote sensing, Chinese agricultural science, vol. 5, pp. 87-88, 2017.

[7] Y. Lin, N. Wei, Hyperspectral characteristics and soil moisture content inversion of spring maize under drought conditions, Journal of ecology, vol. 5, pp. 23-24, 2016.

[8] J. Wu, L.J. Tan and K.R. Deng, Remote sensing estimation of water content in maize canopy based on optimization index, Journal of Hunan agricultural university (self-science edition), vol. 6, pp. 68-69, 2015.

[9] J.F. Jian, H.Ch. He and X.F. Wang, Overview of remote sensing monitoring of plant diseases and insect pests, Bulletin of surveying and mapping, vol. 9, pp. 24-28, 2018.

[10] H.B. Qiao, Y. Shi and W. Guo, Monitoring of wheat take-all disease by near-earth imaging hyperspectral remote sensing, Journal of plant protection, vol. 3, pp. 475-476,2015. 